\title{
Intersubunit Concerted Cooperative and cis-Type Mechanisms Modulate Allosteric Gating in Two-Pore- Domain Potassium Channel TREK-2
}

\author{
Ren-Gong Zhuo ${ }^{1 \dagger}$, Peng Peng ${ }^{1,2}$, Xiao-Yan Liu ${ }^{1}$, Hai-Tao Yan ${ }^{1}$, Jiang-Ping X ${ }^{3}$, \\ Jian-Quan Zheng ${ }^{1}$, Xiao-Li Wei ${ }^{1 *}$ and Xiao-Yun Ma ${ }^{1 *}$ \\ ${ }^{1}$ State Key Laboratory of Toxicology and Medical Countermeasures, Beijing Key Laboratory of Neuropsychopharmacology, \\ Department of Biochemical Pharmacology, Beijing Institute of Pharmacology and Toxicology, Beijing, China, ${ }^{2}$ Anesthesia and \\ Operation Center, PLA General Hospital, Beijing, China, ${ }^{3}$ Department of Pharmacology, School of Pharmaceutical Sciences, \\ Southern Medical University, Guangzhou, China
}

\section{OPEN ACCESS}

Edited by:

Dieter Wicher

Max Planck Institute for Chemical Ecology, Germany

Reviewed by:

Sushil K. Jha,

Jawaharlal Nehru University, India Mathew V. Jones, University of Wisconsin-Madison,

*Correspondence: Xiao-Li Wei weix@@bmi.ac.cn; Xiao-Yun Ma maxy@bmi.ac.cn

tPresent address:

Ren-Gong Zhuo, Department of Traditional Chinese Medicine, Medical College, Xiamen University, Xiamen,

China

Received: 01 February 2016 Accepted: 29 April 2016

Published: 13 May 2016

Citation:

Zhuo R-G, Peng P, Liu X-Y, Yan H-T, $X u J$ - $P$, Zheng J-Q, Wei $X-L$ and Ma X-Y (2016) Intersubunit Concerted Cooperative and cis-Type Mechanisms Modulate Allosteric Gating in Two-Pore-Domain Potassium Channel TREK-2. Front. Cell. Neurosci. 10:127. doi: 10.3389/fncel.2016.00127
In response to diverse stimuli, two-pore-domain potassium channel TREK-2 regulates cellular excitability, and hence plays a key role in mediating neuropathic pain, mood disorders and ischemia through. Although more and more input modalities are found to achieve their modulations via acting on the channel, the potential role of subunit interaction in these modulations remains to be explored. In the current study, the deletion (lack of proximal C-terminus, $\Delta \mathrm{pCt}$ ) or point mutation (G312A) was introduced into TREK-2 subunits to limit $\mathrm{K}^{+}$conductance and used to report subunit stoichiometry. The constructs were then combined with wild type (WT) subunit to produce concatenated dimers with defined composition, and the gating kinetics of these channels to 2-Aminoethoxydiphenyl borate (2-APB) and extracellular $\mathrm{pH}\left(\mathrm{pH}_{0}\right)$ were characterized. Our results show that combination of WT and $\Delta \mathrm{pCt} / \mathrm{G} 312 \mathrm{~A}$ subunits reserves similar gating properties to that of WT dimmers, suggesting that the WT subunit exerts dominant and positive effects on the mutated one, and thus the two subunits controls channel gating via a concerted cooperative manner. Further introduction of $\Delta \mathrm{pCt}$ into the latter subunit of heterodimeric channel G312A-WT or G312A-G312A attenuated their sensitivity to 2-APB and $\mathrm{pH}_{0}$ alkalization, implicating that these signals were transduced by a cis-type mechanism. Together, our findings elucidate the mechanisms for how the two subunits control the pore gating of TREK-2, in which both intersubunit concerted cooperative and cis-type manners modulate the allosteric regulations induced by 2-APB and $\mathrm{pH}_{\circ}$ alkalization.

Keywords: TREK-2, two-pore domain potassium channel, 2-APB, allosteric regulation, intersubunit interaction, concatenated dimer

\section{INTRODUCTION}

Two-pore domain $\mathrm{K}^{+}(\mathrm{K} 2 \mathrm{P})$ channels, the last discovered $\mathrm{K}^{+}$channel family, are major contributors to background $\mathrm{K}^{+}$conductance by producing "leak" currents, and play a predominant role in stabilizing resting membrane potential as well as regulating cellular excitability. Up to now, 15 members, which are divided into six subgroups, have been found in mammals. TREK-2 (TWIK related $\mathrm{K}^{+}$channel, K2P10.1), along with TREK-1 and 
TRAAK (TWIK-related arachidonic acid-stimulated $\mathrm{K}^{+}$ channel), belongs to the TREK/TRAAK K2P subfamily (Enyedi and Czirják, 2010). In addition to central nervous system (Lesage et al., 2000), the channel is also highly expressed in dorsal root ganglia (DRG) and trigeminal ganglia (Kang and Kim, 2006; Yamamoto et al., 2009; Acosta et al., 2014). In DRG C-fiber nociceptors, the functions of TREK-2 are involved in hyperpolarizing membrane potential and limiting spontaneous pain (Kang and Kim, 2006; Acosta et al., 2014). Evidence from knockout mice show the channel is associated with thermosensation and neuropathic pain (Pereira et al., 2014). Activating TREK-2 leads to GABAnergic and noradrenergic inhibition of neuronal excitability in the entorhinal cortex, which is regarded as the gateway to the hippocampus and thus is essential for learning and memory (Deng et al., 2009; Xiao et al., 2009). In response to ischemia, TREK-2 expression is up-regulated in the astrocytic membrane (Rivera-Pagán et al., 2015), cortical and hippocampal neurons (Li et al., 2005). Therefore, TREK-2 has been implicated to be a new potential therapeutic target for treating neuropathic pain, mood disorders and ischemia.

Similar with other K2Ps, single TREK-2 molecule is composed of four helical transmembrane domains (M1-M4) and two pore-forming domains (P-domain). Classical $\mathrm{K}^{+}$channels need to tetramize to form the canonically four-fold pore, whereas dimerization is sufficient for K2Ps, due to their two $\mathrm{P}$-domains are fused into one molecule subunit. Thus, the pore of $\mathrm{K} 2 \mathrm{Ps}$ is assembled as a pseudotetrameric structure compared with classic $\mathrm{K}^{+}$channels. There arranges an inner gate (the lower parts of M2 and M4) and a selectivity filter (SF) gate along the ion pathway (pore region) from outside to inside. As a thermo- and mechano-gated $\mathrm{K}^{+}$channel, TREK-2 is also regulated by lysophospholipids, polyunsaturated fatty acids, G-protein coupled receptors, extra- or intracellular $\mathrm{pH}$, and a range of clinically useful drugs including volatile anesthetics (Bang et al., 2000; Lesage et al., 2000; Deng et al., 2009; Sandoz et al., 2009; Xiao et al., 2009, 2014). It has been reported that some stimuli, such as $\mathrm{Ba}^{2+}$, can block $\mathrm{K}^{+}$ efflux by binding to the pore directly (Zhuo et al., 2015b). However, most of stimuli control the velocity or quality of $\mathrm{K}^{+}$efflux primarily by coupling with the movements of pore region, a mechanism known as allosteric regulations. This mechanism is the most common and effective strategy to control protein activity, especially in allowing signal transmission over a long distance (Perutz, 1989). In multisubunit channels, intersubunit cooperativity might occur during conformational transition in the presence of stimuli or voltage gating. In voltage-dependent $\mathrm{K}^{+}$channels $(\mathrm{Kv})$, it has been established that voltage opens the pore via a concerted cooperative or sequential gating transitions in the tetrameric complex (Tytgat and Hess, 1992; Pathak et al., 2005; Zandany et al., 2008; Gagnon and Bezanilla, 2009, 2010; Yifrach et al., 2009; Meisel et al., 2012; Thomson et al., 2014; Wu et al., 2014a,b). However, no such progress is made in K2Ps. Although more and more signals have been found to act on TREK-2 channel to exert their biological functions, little is known about the putative mechanism of intersubunit interaction, and the manner of signal transduction along single subunit during gating.

Our previous studies have demonstrated that 2-Aminoethoxydiphenyl borate (2-APB), a membranepermeable compound, stimulates the activity of TREK-2 via acting on its cytosolic proximal C-terminus (pCt; Zhuo et al., 2015a), and opens the pore regardless of the original conformation of SF gate (Zhuo et al., 2016). This allosteric transduction (named as "2-APB pathway") is initiated from cytosolic $\mathrm{Ct}$ and propagated to $\mathrm{SF}$ gate. In addition, changes of extracelluar $\mathrm{pH}\left(\Delta \mathrm{pH}_{\mathrm{o}}\right)$ induce the conformational transition of SF gate in K2Ps via an external sensor (Clarke et al., 2008; Cohen et al., 2008; Stansfeld et al., 2008; Niemeyer et al., 2010; Ma et al., 2011; Zúñiga et al., 2011), including TREK-2 (Sandoz et al., 2009; Zhuo et al., 2015a). Such transition in TREK-2 is also facilitated by the pCt domain in TREK-2 (Zhuo et al., 2016). This transmission is evoked by the charge change of sensor and propagated such stimuli to SF gate, and ultimately to intracellular pCt domain, a process called as " $\mathrm{pH}_{\mathrm{o}}$ pathway". These two pathways (2-APB pathway and $\mathrm{pH}_{\mathrm{o}}$ pathway) represent bidirectional and long-distance allosteric couplings between SF gate and pCt domain, and thus provide two facile models to study intersubunit interaction in TREK-2. Except for the pCt domain, the glycine hinge of M4 (G312) has been found to be another essential gating element, which controls the two pathways bidirectionally (Zhuo et al., 2016). Here, to determine the nature of signal transduction along single or double subunit, we constructed a concatenated dimeric TREK-2 channel and incorporated separately or simultaneously the two negative gating mutations, $\Delta \mathrm{pCt}$ and G312A, into single or double subunits. We investigated the effects of these mutations or deletions in the context of tandem-linked dimers on both 2-APB pathway and $\mathrm{pH}_{\mathrm{o}}$ pathways, and found that concerted cooperative intersubunit interaction and cis-type transduction along single subunit underlie the allosteric regulations of TREK-2 channels.

\section{MATERIALS AND METHODS}

\section{Molecular Biology}

Human wild type (WT) forms of TREK-2 (NCBI reference sequence NM_138318)-expressing vector (pGH19-TREK-2) was constructed using Bgl II and Hind III sites. Point mutations and deletions were engineered using the MutanBEST kit (TaKaRa, Dalian, China)-guided high-fidelity PCR. The concatenated dimeric constructs were built to contain a different combination of WT and/or mutant (or deletant) TREK-2 cDNAs in defined position. The two consecutive subunits were connected by a flexible linker encoding the AAAGSGGSGGSTGGSSGSSGS sequence (Bagriantsev et al., 2012) with a little modification. A Sal I site was designed in the middle of the linker to facilitate cloning. One mutation (G312A) and two deletions (residues from W326 to A374 or from I323 to N543 were deleted in $\Delta \mathrm{pCt}$ and $\Delta \mathrm{Ct}$, respectively) were used in this 
study. G312 is located in the middle of M4 domain, and pCt is covalently linked with $\mathrm{M} 4$ and resides on the cytoplasmic side. For all the monomer (WT, G312A, $\Delta \mathrm{pCt}$ and $\Delta \mathrm{Ct}$ ) used to make dimers, $\mathrm{Bgl}$ II/Sal I was used to clone the former subunit, and Sal I/Hind III to the latter subunit. The name of concatenated dimers was defined as the relative position of monomeric channels. For example, WT-G312A represented that the first subunit was WT channel, and the second one was G312A channel. To investigate whether the flexible linker between monomers was degraded in mammalian cells during processing of the protein, either prior to or after membrane insertion, the concatenated WT dimeric construct (WT-WT) was also inserted into the pEGFPN1 vector (Clontech Laboratories, Mountain view, CA, USA) by using Bgl II and Sal I. All the constructs were confirmed by DNA sequencing. All the pGH19-based Plasmids were linearized by Xho I before in vitro transcription. cRNA was synthesized using the RiboMAX ${ }^{\mathrm{TM}}$ Large Scale RNA Production Systems (Promega, Madison, WI, USA) kit.

\section{Channel Expression in Xenopus Oocytes}

Procedures used for harvesting oocytes from Xenopus laevis were approved by the Animal Care and Use Committee at Beijing Institute of Pharmacology and Toxicology. After excised from Xenopus laevis, pieces of ovarian lobes were subjected to collagenase (Sigma Aldrich, St Louis, MO, USA) digestion. Stage $\mathrm{V}$ or VI oocytes were selected and cRNA $(0.1 \sim 10 \mathrm{ng} / 46 \mathrm{nl}$ for each oocyte) was microinjected. Injected cells were incubated at $18^{\circ} \mathrm{C}$ in $\mathrm{ND} 96$ medium $(96 \mathrm{mM} \mathrm{NaCl}, 2 \mathrm{mM} \mathrm{KCl}, 1.8 \mathrm{mM}$ $\mathrm{CaCl}_{2}, 1 \mathrm{mM} \mathrm{MgCl} 2,10 \mathrm{mM}$ HEPES, $5 \mathrm{mM}$ pyruvate, $100 \mathrm{mg} / \mathrm{ml}$ gentamycin, $\mathrm{pH}$ 7.2).

\section{Electrophysiology}

Whole-cell currents were measured 1-3 days after injection, and amplified using an Axoclamp2B amplifier (Axon Instruments, Union City, CA, USA) in two-electrode voltage clamp (TEVC) mode. Microelectrodes were pulled with a tip resistance of 0.1-1 M $\Omega$ when filled with $3 \mathrm{M} \mathrm{KCl}$. Data were sampled at $2 \mathrm{kHz}$ and filtered at $0.5 \mathrm{kHz}$ with Clampex 10.0 software (Axon Instruments). Recordings were performed under standard, physiological extracellular solution $(5 \mathrm{mM} \mathrm{KCl}, 93 \mathrm{mM} \mathrm{NaCl}$, $1 \mathrm{mM} \mathrm{MgCl}_{2}, 1.8 \mathrm{mM} \mathrm{CaCl}_{2}, 5 \mathrm{mM}$ HEPES, pH 7.4, adjusted with $\mathrm{NaOH}$ ), and constant perfusion at room temperature. TREK-2 currents were elicited by continuous voltage-ramps from -120 to $+60 \mathrm{mV}$ from a holding potential of $-80 \mathrm{mV}$, with $2 \mathrm{~s}$ in duration (current-voltage relationship, I-V curve). The currents of TREK-2 undergo obvious "run-up" at the beginning of recording, thus, each measurement was performed after the stabilization was reached (about $20 \mathrm{~min}$ ) before applying stimulus. 2-APB (Promega, Madison, WI, USA) was diluted with the standard solution freshly.

\section{Cell Culture and Western Blotting Analysis}

HEK 293 cells were cultured in DMEM supplemented with 10\% fetal bovine serum and $2 \mathrm{mM} \mathrm{L}$-glutamine and held at $37^{\circ} \mathrm{C}$ in humidified air with $5 \% \mathrm{CO}_{2}$. Constructs encoding GFP (vector),
TREK-2-GFP (monomer) and WT-WT-GFP (tandem-linked dimer) were transiently transfected into HEK 293 cells using Lipofectamine 2000 (Invitrogen, Carlsbad, CA, USA). Two days after transfection, the cells were harvested. Protein extracts were prepared by solubilizing in buffer ( $\mathrm{pH}$ 7.4, $50 \mathrm{mM}$ Tris, $270 \mathrm{mM}$ $\mathrm{NaCl}, 1 \%$ Triton $\mathrm{X}-100$ ) for $1 \mathrm{~h}$ and clarified by centrifugation at $12,000 \times \mathrm{g}$ for $30 \mathrm{~min}$. Oocytes were injected with $10 \mathrm{ng}$ cRNA, and then they were lyzed after $48 \mathrm{~h}$ in a cold buffer of $150 \mathrm{mM} \mathrm{NaCl}, 1.06 \mathrm{mM} \mathrm{KH} \mathrm{KH}_{2} \mathrm{PO}, 2.07 \mathrm{mM} \mathrm{Na}_{2} \mathrm{HPO}_{4}$, $1 \%$ Triton X-100, pH 7.4 supplemented with antiproteases and clarified by centrifugation at $20,000 \times \mathrm{g}$ for $15 \mathrm{~min}$. The above lysates were subjected to SDS-PAGE on $10 \%$ gel and wet-transferred onto a PVDF membrane. The lysates were hybridized with anti-TREK-2 antibody (Abgent, San Diego, 1:200) or anti-GFP (Cell Signaling Technology, Danvers, MA, USA, 1:1000) for oocytes and HEK293 in Western blotting, respectively. Actin (anti-actin antibody was purchased from ZSGB-BIO, China, 1:5000) was used as loading control. Reaction bands were visualized by incubating the blots with appropriate fluorescence-tagged secondary antibodies (Lincoln, NE, USA, $1: 15,000)$.

\section{Data Analysis}

Voltage clamp data were analyzed with origin 8.0 (OriginLab Corporation, Northampton, MA, USA) and GraphPad prism version 5.0 (GraphPad Software Inc., La Jolla, CA, USA). The currents recorded at $0 \mathrm{mV}$ were used to calculate all the ratios, which were presented as mean \pm SEM, and reflected $n=5$ or more from at least two batches of oocytes. Activation ratio (AR) was used to observe the stimulatory effects of externally applied 2-APB on TREK-2. AR was calculated from $\mathrm{I}_{2-\mathrm{APB}} / \mathrm{I}_{\mathrm{O}}$, where $\mathrm{I}_{2-\mathrm{APB}}$ represented the currents in the presence of 2-APB, and $I_{O}$ was the baseline, stabilized currents in the absence of 2-APB, and was plotted as function of the concentration of $2-\mathrm{APB}\left([2-\mathrm{APB}]_{\mathrm{o}}\right)$. To estimate the inhibitory effects of extracellular alkalization, inhibition ratio (IR) was calculated from $1-\mathrm{I} / \mathrm{I}_{\mathrm{pH} 6.5}$, where I represented the currents in a specific $\mathrm{pH}$, and $\mathrm{I}_{\mathrm{pH} 6.5}$ was the stabilized currents recorded at $\mathrm{pH} 6.5$, and was plotted as a function of different $\mathrm{pH}\left(\mathrm{pH}_{\mathrm{o}}\right)$. The resulted ratios were fitted to the Hill equation. Statistical differences were tested by using unpaired Student's $t$ test where appropriate. Differences were considered significant if $p<0.05$.

\section{RESULTS}

\section{TREK-2 Concatenated Dimer Reserves Similar Gating Properties as its Monomer}

Mature TREK-2 channel forms as homodimers, so mutations or deletions of the channel can involve two subunits simultaneously. To perform a quantitative study of intersubunit or intrasubunit interaction, a strategy that employs concatenated dimer was adopted. TREK-2 tandem constructs, in which the two subunits are connected by a 21-amino-acid flexible linker (Bagriantsev et al., 2012; WT-WT, Figure 1A) were produced and expressed in Xenopus oocytes. To investigate whether the tandem channels 

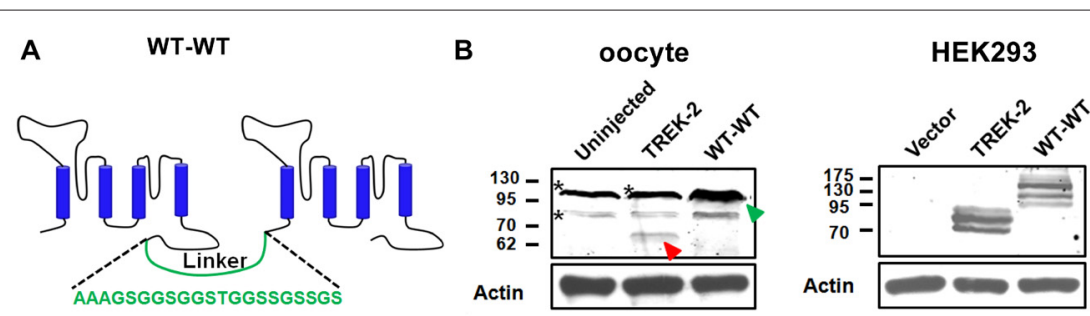

C

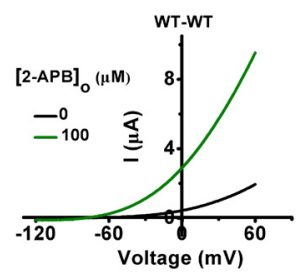

E

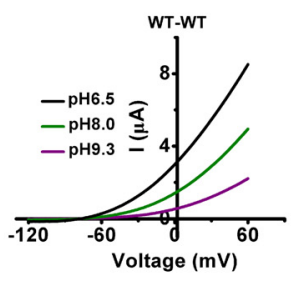

D

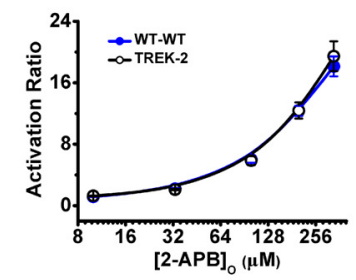

$\mathbf{F}$

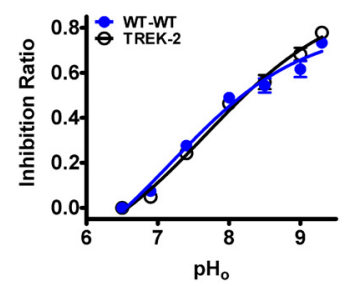

FIGURE 1 | Concatenated wild type (WT) TREK-2 dimers (WT-WT) functions similarly with their monomeric WT channels (TREK-2). (A) Diagram of concatenated TREK-2 dimer and the sequence of linker indicated. (B) Validation of TREK-2 and WT-WT proteins was tested by western blotting. cDNA encoding TREK-2 or WT-WT was injected into oocytes. The monomeric TREK-2 was indicated with red arrow, and the dimeric one with green arrow. *Indicates the nonspecific bands detected by the anti-TREK-2 primary antibody in oocytes (left panel). Constructs harboring GFP-tagged TREK-2 or WT-WT was transfected into HEK293 cells (right panel). (C) Exemplar current-voltage recordings from oocytes expressing the WT-WT channels in the presence of 100 $\mu$ M 2-APB. The currents were elicited by continuous voltage-ramps from -120 to $+60 \mathrm{mV}$ from holding potential of $-80 \mathrm{mV}$, with $2 \mathrm{~s}$ in duration. (D) Comparative analysis of the 2 -APB evoked activation curves between TREK-2 and WT-WT channels. (E) Exemplar current-voltage recordings from oocytes expressing WT-WT channels as pH ${ }_{0}$ transitions among 6.5, 8.0 and 9.3. (F) Concentration dependence of Inhibition ratio (IR) upon extracellular alkalization for TREK-2 and WT-WT channels.

are properly expressed, we firstly detected the expressed proteins by monomeric (TREK-2) and concatenated dimeric constructs (WT-WT) in Xenopus oocytes using western blotting. As shown in Figure 1B (left panel), the monomeric construct produces one specific band corresponding to the approximate size of mature monomer (red arrow). Notably, two nonspecific bands were also detected by the anti-TREK-2 primary antibody in oocytes (asterisks). The WT-WT construct produces a specific band (green arrow, overlapped with a nonspecific band) corresponding with dimeric TREK-2. No monomeric signal was detected by In WT-WT construct-injected oocytes. To rule out the nonspecific expression, we further assessed their expressions in HEK293 cells by transfecting GFP-tagged monomeric and tandem-linked vectors. As shown in Figure 1B (right panel), distinct monomeric or dimeric bands were observed at the predicted molecular mass in TREK-2- or WT-WT-transfected group, respectively, but no monomeric signals were found in WT-WT group. Notably, no immunoreactivity was detected in the pEGFPN1-transfected cells (vector). These data indicate that the concatamers remain intact and the linker is not susceptible to degradation, and the WT-WT constructs can be used for further investigations.
To investigate the gating properties of the tandem-linked dimer, 2-APB response of the channel was investigated and compared with that of monomeric TREK-2. As the currentvoltage relationships ( $\mathrm{I}-\mathrm{V}$ curves) shown in Figure 1C, the currents recorded from WT-WT channels was radically stimulated by $100 \mu \mathrm{M} 2$-APB. The activation ratio (AR, measured using the currents at $0 \mathrm{mV}$ ) values were plotted against their 2-APB concentrations, and results show that the activating effects of 2-APB on WT-WT channels was similar with that of monomeric TREK-2 (Figure 1D). The maximal AR ( $\mathrm{AR}_{\max }$, produced by $333 \mu \mathrm{M} 2-\mathrm{APB}$, unless otherwise specified) produced by TREK-2 monomer and WT-WT tandem-linked dimer was $19.46 \pm 1.94$ and $18.13 \pm 1.30$, respectively (Table 1).

To further evaluate the gating properties of the concatenated dimer, we also measured its response to extracellular alkalization. When the extracellular $\mathrm{pH}\left(\mathrm{pH}_{\mathrm{o}}\right)$ was switched from 6.5 to 8.0 or 9.3 , the currents of WT-WT channels was drastically inhibited (Figure 1E). Importantly, the dose response curves (Figure 1F) demonstrating that the IRs against their $\mathrm{pH}_{\mathrm{o}}$ clearly revealed that the tandemly linked dimer was inhibited by extracellular alkalization in a similar degree with monomer. The maximal IR ( $\mathrm{IR}_{\max }$, produced by $\mathrm{pH}$ 9.3) for monomeric 
TABLE 1 | Response of TREK-2 tandem-linked channels and mutants to 2-APB and $\mathrm{pH}_{\mathrm{o}}$ changes

\begin{tabular}{lccccc}
\hline & \multicolumn{2}{c}{ 2-APB response } & & \multicolumn{2}{c}{$\mathbf{p H}_{\mathbf{o}}$ response } \\
\cline { 2 - 3 } Channel & $\mathbf{A R}^{\mathbf{a}}{ }_{\text {max }}$ & $\mathbf{n}^{\mathbf{b}}$ & & $\mathbf{I R}^{\mathbf{c}}{ }_{\max }$ & $\mathbf{n}^{\mathbf{b}}$ \\
\hline WT-WT & $18.13 \pm 1.30$ & $8-13$ & & $0.73 \pm 0.02$ & $8-9$ \\
$\Delta \mathrm{pCt}-\Delta \mathrm{pCt}$ & $4.34 \pm 0.10$ & 7 & & $0.46 \pm 0.01$ & 7 \\
WT- $\Delta \mathrm{pCt}$ & $15.50 \pm 0.74$ & $5-7$ & & $0.79 \pm 0.01$ & 5 \\
$\Delta \mathrm{pCt-WT}$ & $17.37 \pm 1.56$ & $5-6$ & & $0.77 \pm 0.02$ & 6 \\
WT- $\Delta$ Ct & $18.15 \pm 2.42$ & $5-7$ & & $0.76 \pm 0.03$ & $7-9$ \\
$\Delta$ Ct-WT & $16.78 \pm 1.50$ & $6-7$ & & $0.81 \pm 0.02$ & $6-10$ \\
WT-G312A & $17.16 \pm 1.00$ & $6-8$ & & $0.76 \pm 0.04$ & $6-7$ \\
G312A-WT & $16.84 \pm 2.00$ & $6-7$ & & $0.79 \pm 0.01$ & $6-7$ \\
G312A-G312A & $7.80 \pm 0.50$ & $5-7$ & & $0.23 \pm 0.02$ & $5-7$ \\
G312A- $\Delta$ pCt & $5.32 \pm 0.46$ & 7 & & $0.43 \pm 0.03$ & $11-12$ \\
G312A-G312A/ $\Delta$ pCt & $3.36 \pm 0.37$ & $7-8$ & & $0.12 \pm 0.05$ & $7-12$
\end{tabular}

aThe maximal concentrations of 2-APB used in these curves is $333 \mu \mathrm{M}$ for these channels, except for $\Delta p C t-\Delta p C t(800 \mu \mathrm{M}) .{ }^{b}$ The number of oocytes used for each point in concentration response curves. ${ }^{c}$ The maximal inhibition produced by $\mathrm{pH}$.3.3.

TREK-2 and WT-WT channels was $0.78 \pm 0.02$ and $0.73 \pm 0.02$, respectively (Table 1). Taken together, these data suggest that the tandem-linked strategy does not influence the gating properties of TREK-2 channels. Thus, this method was further used to investigate how the intersubunit and intrasubunit interplay.

\section{The Proximal C-Terminus Controls the 2-APB Pathway in a Concerted Cooperative Manner Between Subunits}

Our previous study have identified that the cytoplasmic pCt (from W326 to A374, the relative position was illustrated in Figure 2A) plays an essential role in gating TREK-2 (Zhuo et al., 2016), so we further used this negative gating mutation to investigate how these two subunits may interact with each other. $\Delta \mathrm{pCt}$ was constructed as either the first subunit $(\Delta \mathrm{pCt}-\mathrm{WT})$ or the second subunit (WT- $\Delta \mathrm{pCt}$ ). The response of these heterodimers to 2-APB were investigated and compared with WT-WT and $\Delta \mathrm{pCt}-\Delta \mathrm{pCt}$ homodimers.

When the gating of one subunit in TREK-2 dimer does not alter the gating kinetics of another one, the heterodimers should respond to stimuli in a linear combination for their two homodimers (such as WT-WT and $\Delta \mathrm{pCt}-\Delta \mathrm{pCt}$ in our study), and such interactive pattern belongs to independent or sequential manner. If the response of heterodimers to stimuli is similar with those of homodimers, the gating between subunits is compatible with concerted cooperative fashion (Zandany et al., 2008; Tombola et al., 2010; Meisel et al., 2012; Thomson et al., 2014). As shown in Figure 2B, $100 \mu \mathrm{M}$ 2-APB strongly stimulated the currents of WT- $\Delta \mathrm{pCt}$, while the activity of $\Delta \mathrm{pCt}-\Delta \mathrm{pCt}$ was only slightly stimulated. Comparison of the concentration-dependent curves of the four channels further indicated that the 2-APB responses of both WT- $\Delta \mathrm{pCt}$ and $\Delta \mathrm{pCt}-\mathrm{WT}$ channels were more similar to that of WT-WT than to that of $\Delta \mathrm{pCt}-\Delta \mathrm{pCt}$ (Figure 2C). The $\mathrm{AR}_{\max }$ for WT- $\Delta \mathrm{pCt}$ and $\Delta$ pCt-WT was $15.50 \pm 0.74$ and $17.37 \pm 1.56$, respectively. However, the ratio of $\Delta \mathrm{pCt}-\Delta \mathrm{pCt}$ was only $4.34 \pm 0.10$ even when the concentration of 2 -APB was enhanced to $800 \mu \mathrm{M}$ (Table 1).

Our previous study has also revealed that the full $\mathrm{Ct}$ regulates the gating of TREK-2 in a similar manner with pCt (Zhuo et al., 2016), so here we measured 2-APB responses of $\Delta \mathrm{Ct}$ deletant incorporated heterodimers (WT- $\Delta \mathrm{Ct}$ and $\Delta \mathrm{Ct}-\mathrm{WT}$ ). Consistent with the results from $\Delta \mathrm{pCt}$ heterodimer, both WT- $\Delta \mathrm{Ct}$ and $\Delta \mathrm{Ct}-\mathrm{WT}$ exhibited similar sensitivity to $2-\mathrm{APB}$ as that of WT-WT channels (Figure 2D and Table 1). Taken together, the WT subunit exerts strong, positive effects on the $\Delta \mathrm{Ct}$ or $\Delta \mathrm{pCt}$ subunit in
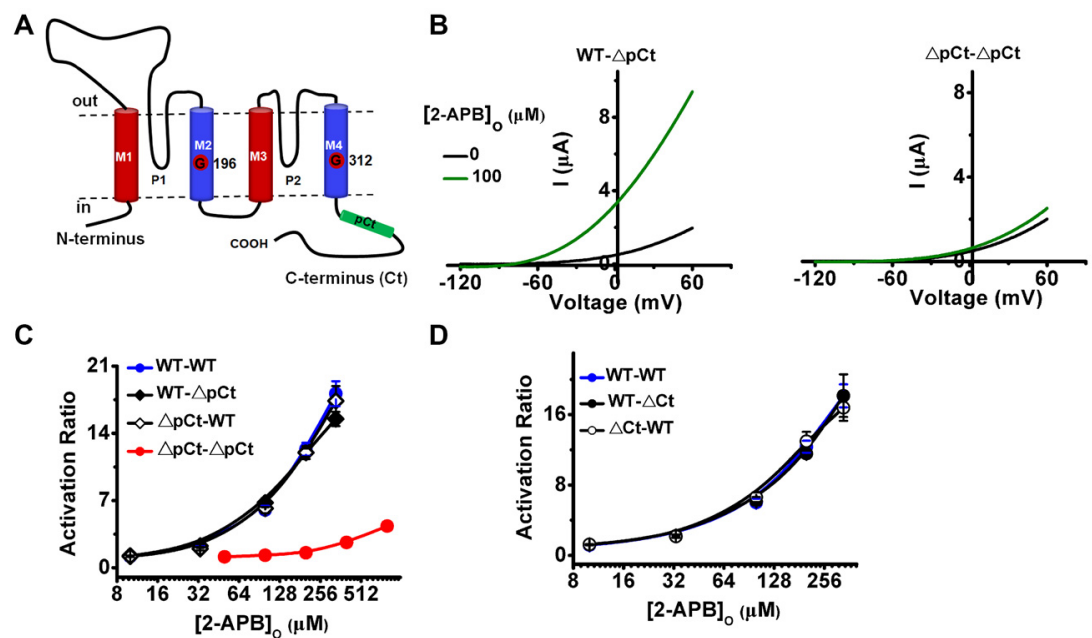

D

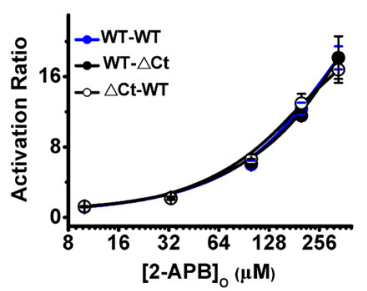

FIGURE 2 | The effects of incorporation of $\Delta \mathrm{pCt}$ or $\Delta \mathrm{Ct}$ on the gating processes induced by 2-APB. (A) Diagram of a single TREK-2 subunit, transmembrane segments 1 4 (M1 M4), N-terminus, the proximal C-terminus (pCt), pore domain 1 (P1) and 2 (P2), glycine hinge of M2 (G196) and M4 (G312) are indicated. (B) Exemplar current-voltage recordings from oocytes expressing the indicated channels in the presence of $100 \mu \mathrm{M} 2-\mathrm{APB}$. (C,D) Comparative analysis of the 2-APB evoked activation curves for the indicated channels. Due to the lower sensitivity of $\Delta \mathrm{pCt}-\Delta \mathrm{pCt}$ to 2-APB, higher concentrations were used in its curve. 
A

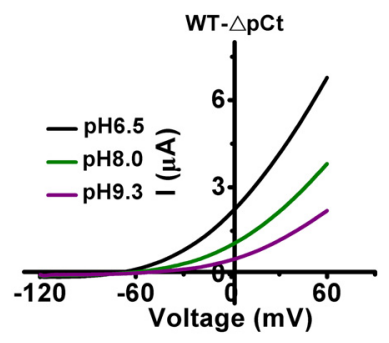

B

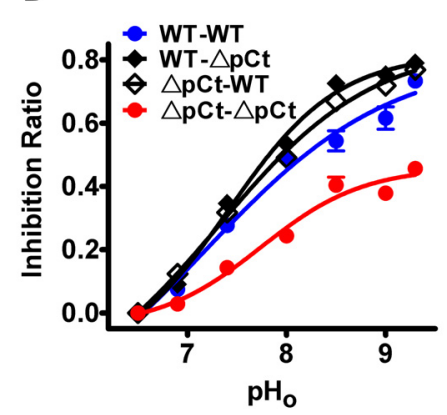

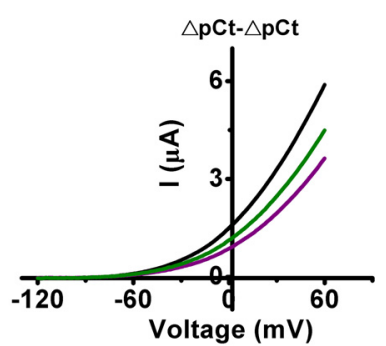

C

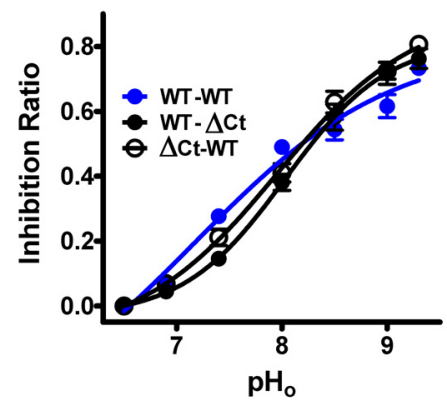

FIGURE 3 | The effects of incorporation of $\Delta \mathrm{pCt}$ or $\Delta \mathrm{Ct}$ on the gating processes induced by extracellular alkalization. (A) Exemplar current-voltage recordings from oocytes expressing the indicated channels as $\mathrm{pH}_{0}$ transitions among 6.5, 8.0 and 9.3. (B,C) $\mathrm{IR}$ of indicated channels to $\mathrm{pH}_{0}$ fluctuation.

response to 2 - APB stimulation, suggesting that the concerted intersubunit cooperativity gates TREK-2 channels at 2 -APB pathway.

\section{The Proximal C-Terminus Regulates the Extracellular Alkalization-Induced Gating via an Intersubunit Cooperative Manner}

To confirm above conclusion, we also evaluated the $\Delta \mathrm{Ct}$ or $\Delta \mathrm{pC}$-contained heterodimers to $\mathrm{pH}_{\mathrm{o}}$ changes $\left(\mathrm{pH}_{\mathrm{o}}\right.$ pathway). Similar with the situation of 2-APB response, WT- $\Delta$ pCt channels were strongly inhibited by extracellular alkalization, whereas the currents from $\Delta \mathrm{pCt}-\Delta \mathrm{pCt}$ channels were slightly blocked (Figure $\mathbf{3 A}$ ). Furthermore, the responses of WT- $\Delta \mathrm{pCt}\left(\mathrm{IR}_{\max }=0.79 \pm 0.01\right)$ and $\Delta \mathrm{pCt}-\mathrm{WT}$ $\left(\mathrm{IR}_{\max }=0.77 \pm 0.02\right)$, WT- $\Delta \mathrm{Ct}\left(\mathrm{IR}_{\max }=0.76 \pm 0.03\right)$ and $\Delta$ Ct-WT $\left(\mathrm{IR}_{\max }=0.81 \pm 0.02\right)$ to $\mathrm{pH}_{\mathrm{o}}$ changes were more resembled with that of WT-WT channels than that of $\Delta \mathrm{pCt}-\Delta \mathrm{pCt}$ channels (Figures $3 \mathrm{~B}, \mathrm{C}$ and Table 1). Taken together, these results reveal that the heterodimers including pCt or Ct deletant have similar sensitivity in response to $\mathrm{pH}_{\mathrm{o}}$ changes as that of WT-WT channels, suggesting that the $\mathrm{pCt}$ (or $\mathrm{Ct}$ ) domain controls the channel in a concerted intersubunit cooperative manner in the $\mathrm{pH}_{\mathrm{o}}$ pathway.

\section{The Glycine Hinge of M4 (G312) also Controls Channel Gating Cooperatively Between Subunits}

The glycine hinge of M4, G312 (the relative position was illustrated in Figure 2A), is involved in the regulations of both
2-APB and extracellular alkalization (Zhuo et al., 2016). By introducing G312A mutation to either the first or second subunit of WT-WT channels and evaluating their responses to 2-APB and $\Delta \mathrm{pH}_{\mathrm{o}}$, we investigated whether G312 gates TREK-2 in a cooperative or seqential manner between subunits. WT-G312A was activated dramatically by $100 \mu \mathrm{M} 2-\mathrm{APB}$, while the activated degree to G312A-G312A was radically decreased (Figure 4A). The dose response curves of WT-G312A $\left(\mathrm{AR}_{\max }=17.16 \pm 1.00\right)$ and G312A-WT $\left(\mathrm{AR}_{\max }=16.84 \pm 2.00\right)$ were more similar to that of WT-WT channels than to the channel comprising two G312As $\left(\mathrm{AR}_{\max }=7.80 \pm 0.50\right.$; Figure $4 \mathrm{~B}$ and Table 1$)$. In addition, dimerizing G312A with WT subunit exhibited more resembled sensitivity with WT-WT channels to $\Delta \mathrm{pH}_{\mathrm{o}}$ than that of G312A-G312A (Figures 4C,D and Table 1). These results indicate that G312 of the WT subunit exerts positive effects on the mutated one (G312A) during gating processes induced by 2-APB and $\mathrm{pH}_{\mathrm{o}}$, suggesting that the glycine hinge of M4 also gates TREK-2 in a concerted cooperative manner between subunits.

\section{Gly312 and pCt Domain Gate TREK-2 via a cis-Type Mechanism}

Since both Gly312 and pCt control TREK-2 gating cooperatively between subunits in modulating the bidirectional communication between SF gate and pCt domain (Figures 2-4), it is critical to understand how these elements interact with each other during this communication: a cis-type mechanism in which the interaction only occurs within one subunit, or a trans-type mechanism that involves both subunits. To 
A

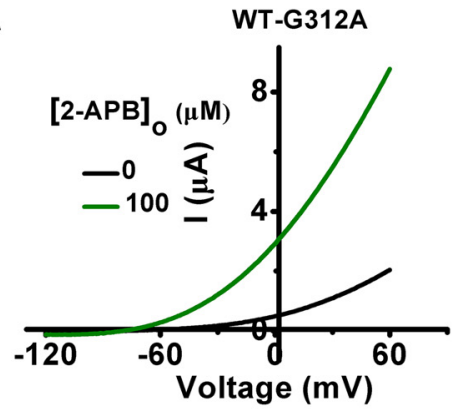

C

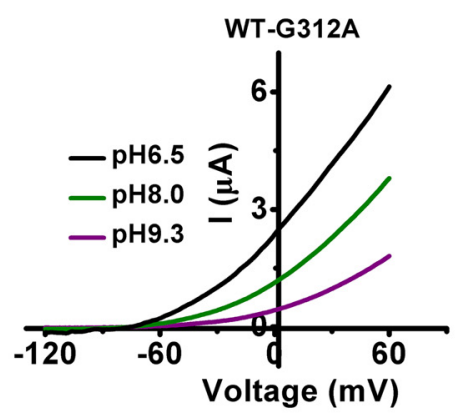

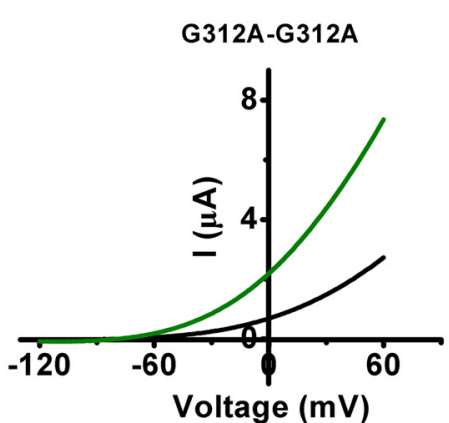

Voltage (mV)

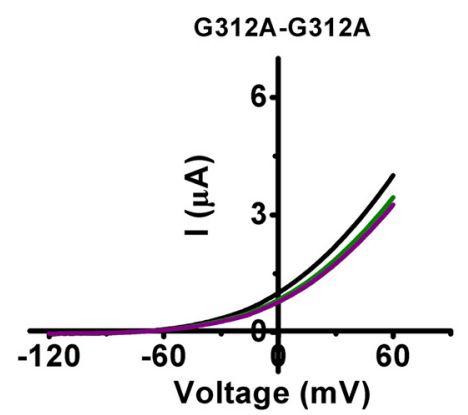

B

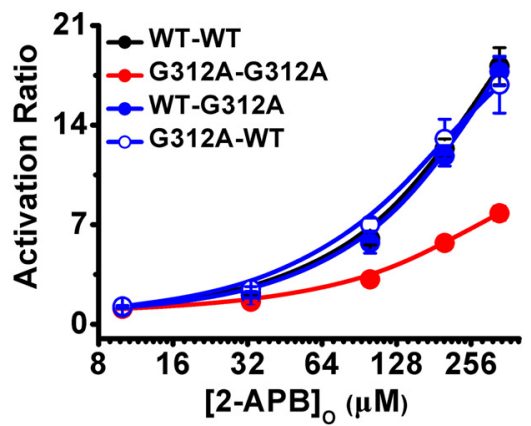

D

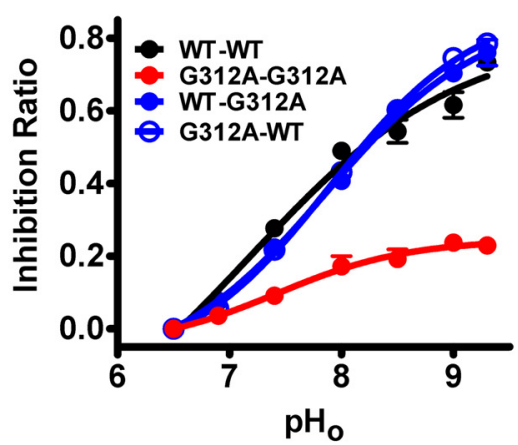

FIGURE 4 | The effects of incorporation of G312A on the gating processes induced by 2-APB and extracellular alkalization. (A) Exemplar current-voltage recordings from oocytes expressing the indicated channels in the presence of $100 \mu \mathrm{M} 2-\mathrm{APB}$. (B) Concentration responses of indicated channels activated by 2-APB. (C) Exemplar current-voltage recordings from oocytes expressing the indicated channels as $\mathrm{pH}_{\mathrm{O}}$ transitions among 6.5, 8.0 and 9.3. (D) Comparative analysis of $\mathrm{pH}_{\mathrm{o}}$-inhibition curves for the indicated channels.

discriminate these two possible mechanisms, we performed mutant cycle analysis. For this purpose, a concatenated dimer with one nonfunctional subunit was required. G312A-WT dimer was chosen because both $2-\mathrm{APB}$ and $\Delta \mathrm{pH}_{\mathrm{o}}$ induced gating pathways were interrupted by G312A mutation in the former subunit. G312A transition and $\Delta \mathrm{pCt}$ deletion were individually or simultaneously introduced into the WT subunit to obtain G312A-G312A (as also described in Figure 4), G312A- $\Delta \mathrm{pCt}$ and G312A-G312A/ $\Delta$ pCt. Then their responses to $2-\mathrm{APB}$ and $\mathrm{pH}_{\mathrm{o}}$ were investigated. If the interaction between $\mathrm{G} 312$ and pCt is trans-type, the pCt of the G312A subunit and the G312 of the $\triangle \mathrm{pCt}$ subunit will rescue the channel function due to their cooperative natures. Accordingly, the effects of 2-APB and $\mathrm{pH}_{\mathrm{o}}$ changes on $\mathrm{G} 312 \mathrm{~A}-\Delta \mathrm{pCt}$ will be similar with those of G312A-WT. Meanwhile, such effects on G312A-G312A/ $\Delta \mathrm{pCt}$ will be analogous with those of G312A-G312A. Nevertheless, the ability of 2 -APB to activate G312A- $\Delta$ pCt was decreased radically compared with $\mathrm{WT}-\Delta \mathrm{pCt}$, and such ability was also decreased in G312A/ $\Delta$ pCt when compared with G312A-G312A (Figure 5A). According to the concentration-dependent curves represented in Figure $5 B$ and Table 1 , the $A R_{\max }$ of G312AWT was $16.84 \pm 2.00$, while the ratio of G312A- $\Delta \mathrm{pCt}$ was decreased to $5.32 \pm 0.46$. Likewise, incorporation of $\Delta \mathrm{pCt}$ into G312A-G312A led to the decrement of $A_{\max }$ from $7.80 \pm 0.50$ in G312A-G312A to $3.36 \pm 0.37$ in G312A-G312A/ $\Delta$ pCt. The $\mathrm{pH}_{\mathrm{o}}$ response of G312A- $\Delta \mathrm{pCt}$ also exhibited blunted gating kinetics (Figures 5C,D) when compared with WT- $\Delta$ pCt. The
$\mathrm{IR}_{\max }$ of WT- $\Delta \mathrm{pCt}$ was $0.79 \pm 0.01$, whereas the ratio of G312A$\Delta \mathrm{pCt}$ was decreased to $0.43 \pm 0.03$ (Table 1). Meanwhile, very similar with the situation in $2-\mathrm{APB}$, introduction of $\Delta \mathrm{pCt}$ into G312A-G312A also resulted in blunted $\mathrm{pH}_{\mathrm{o}}$ response of G312A-G312A/ $\Delta \mathrm{pCt}\left(\mathrm{IR}_{\max }=0.12 \pm 0.05\right)$ when compared with G312A-G312A ( $\left(R_{\max }=0.23 \pm 0.02\right.$; Figures 5C,D). Taken together, further deleting the function of pCt of the WT subunit in G312A-WT or G312A-G312A channel attenuates its sensitivity to 2-APB and $\mathrm{pH}_{\mathrm{o}}$ changes, suggesting that the allosteric regulations triggered by these stimuli occur along one subunit, rather than across both subunits. Namely, G312 and pCt gate TREK-2 channels via a cis-type mechanism.

\section{DISCUSSION}

It has been well established that the activities of K2Ps are tuned by a variety of stimuli from extracellular or intracellular side, nevertheless, how the two subunits respond to these stimuli remains largely unknown. The main purpose of this study was to determine the nature of intersubunit interaction during TREK-2 gating, using concatenated dimeric TREK-2 constructs. By determining the effects of 2-APB and extracellular alkalization on concatenated dimers comprised of WT TREK-2 and loss of function mutants ( $\triangle \mathrm{pCt}$ and $\mathrm{G} 312 \mathrm{~A}$ ), we demonstrate that the two subunits gate channel in a cooperative concerted way, and show that the allosteric regulations evoked by $2-\mathrm{APB}$ and $\mathrm{pH}_{\mathrm{o}}$ changes occur in a cis-type mechanism. 

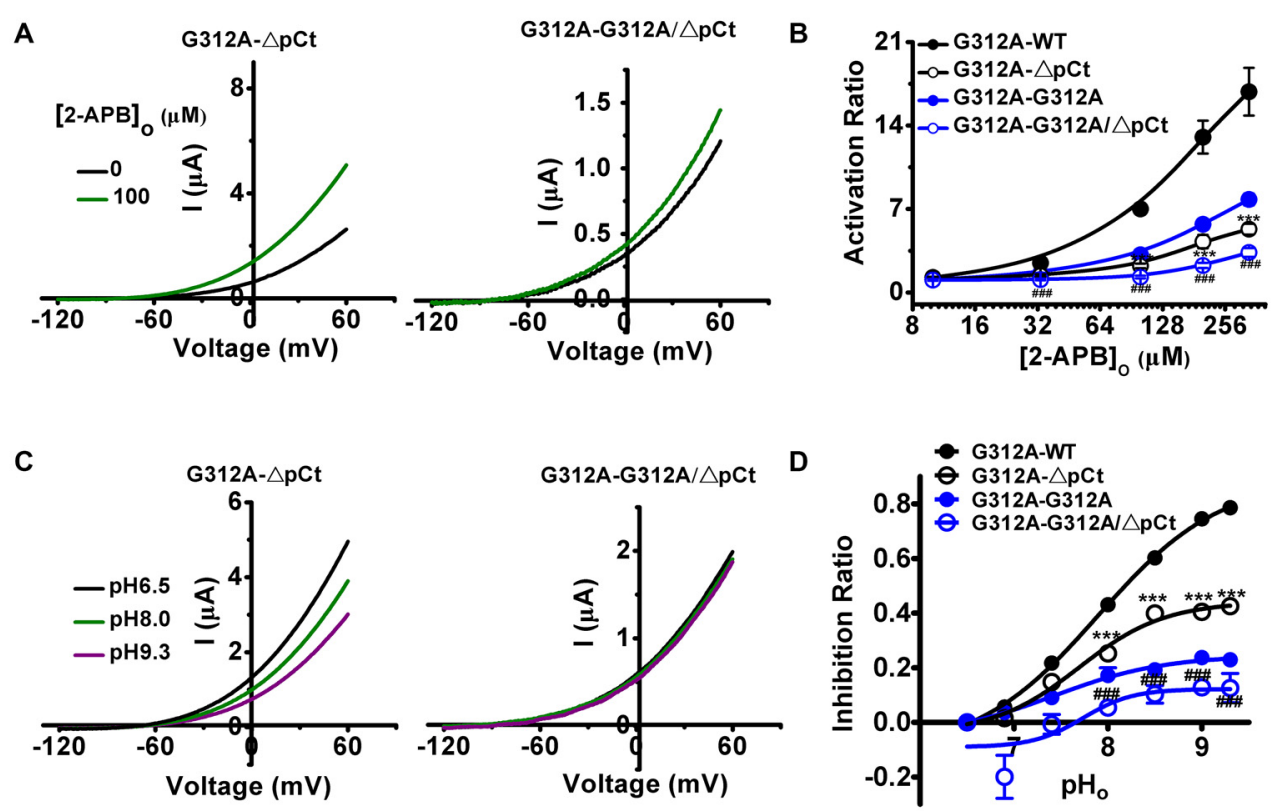

FIGURE 5 | The effects of further introduction of G312A and $\Delta \mathrm{pCt}$, either individually or simultaneously, into the WT subunit of G312A-WT on the gating processes induced by 2-APB and extracellular alkalization. (A) Exemplar current-voltage recordings from oocytes expressing G312A- $\triangle \mathrm{pCt}$ and G312A-G312A $\Delta$ pCt channels in the presence of $100 \mu \mathrm{M} 2$-APB. (B) Concentration responses of indicated channels activated by 2 -APB. ${ }^{\star \star \star} p<0.001$ compared with G312A-WT. \#\# $p<0.05$ compared with G312A-G312A. (C) Exemplar current-voltage recordings from oocytes expressing G312A- $\Delta$ pCt and G312A-G312A/ $\Delta \mathrm{pCt}$ channels as $\mathrm{pH}_{0}$ transitions among 6.5, 8.0 and 9.3. (D) Comparative analysis of $\mathrm{pH}_{\mathrm{o}}$-inhibition curves for the indicated channels. *** $p<0.001$ compared with G312A-WT. \#\#\# $p<0.05$ compared with G312A-G312A.

In homooligomeric proteins, allosteric regulation is often achieved by conformational changes, which may lead to cooperativity between subunits. Such intersubunit cooperativity may be concerted (Monod-Wymann-Changeux model; Monod et al., 1965) or sequential (Koshland-Nemethy-Filmer model; Koshland et al., 1966). To identify the nature of intersubunit cooperativity, researchers generally use concatenated oligomers comprising of WT or mutated subunit. If all the subunits contribute to the gating mechanism, which is manifested by different behavior between homooligomers (either WTs or mutants) and heteroligomers, the cooperativity is sequential. For example, KCNQ1 (Kv7) channels experience sequential gating transitions with independent voltage sensor domain movements, in which the relationship between the shift in the voltage dependence of activation and the number of mutated subunits is linearity (Meisel et al., 2012). Otherwise, if the phenotype of heteroligomers is similar with those of homooligomers, either WT or mutated, the cooperativity between subunits belongs to concerted all-or-none manner. To identify the nature of intersubnit interaction in TREK-2, we constructed tandemly linked dimer, and used loss of function mutation (G312A) or deletion ( $\Delta \mathrm{pCt}$ or $\Delta \mathrm{Ct}$ ) to mark the function of single subunit. Deletion of pCt domain (or the full $\mathrm{Ct}$ ) in a single subunit reserved the normal response to $2-\mathrm{APB}$ and $\mathrm{pH}_{\mathrm{O}}$ changes (Figures 2, 3). In addition, destructing the role of G312 in a single subunit did not affect the sensitivity of TREK-2 to 2-APB and $\mathrm{pH}_{\mathrm{o}}$ changes (Figure 4). These results clearly indicate that at least the glycine hinge of M4 and the pCt domain gate the channel in a intersubunit concerted cooperative manner in both 2-APB and $\mathrm{pH}_{\mathrm{o}}$ pathways. Together with our previous study demonstrating that both pCt and G312 are common gating elements used by these bidirectional pathways (Zhuo et al., 2016), the concerted intersubunit interaction during both pathways further supports that similar gating mechanism may be utilized by inter- and extracellular stimuli in regulating the activity of TREK-2.

In our opinion, the concerted cooperativity could further be classified into two manners: positive and negative. If one single mutated subunit is able to eliminate the associated phenotype of WT channel, the concerted cooperativity is negative, the so-called all-or-none mode. Such manner has been found in the activation gate opening of the shaker $\mathrm{Kv}$ channel (Zandany et al., 2008), the slow deactivation (Thomson et al., 2014), C-type inactivation (Wu et al., 2014a), and drugbinding induced inactivation of human ether-à-go-go-related Gene $\mathrm{K}^{+}$channel 1 (hERG1; Wu et al., 2015b). Otherwise, if one single WT subunit is sufficient to maintain the phenotype of WT channel, the intersubunit interaction is compatible with positive cooperativity, which means the WT subunit exert positive or dominant effects to the mutated ones. The heterodimers comprising of WT and G312A/ $\Delta \mathrm{pCt}$ completely reserve the sensitivity of TREK-2 to $2-\mathrm{APB}$ and $\Delta \mathrm{pH}_{\mathrm{o}}$, suggesting the cooperativity of the two subunits in TREK-2 acts in a positive manner. Such positively concerted cooperativity has only been found in other homomeric channels. In the blocking of $\alpha$-bungarotoxin to homopentameric $\alpha 7$ acetylcholine receptors, a single subunit confers nearly maximal suppression of 
channel opening (daCosta et al., 2015). A voltage-gated proton homodimeric channel, Hv1, utilizes the positive mechanism to harness its voltage sensed gating (Tombola et al., 2010). TREK-1, the closest relative of TREK-2 in K2Ps (Lesage et al., 2000), functions normally when dimerized with loss of function deletant $(\Delta \mathrm{Ct})$ in the presence of either inter- or extracellular stimuli (Sandoz et al., 2012). Single $\mathrm{pH}_{\mathrm{o}}$ sensor of TASK2, another member of K2Ps, is also sufficient to rescue the $\mathrm{pH}_{\mathrm{o}}$ sensitivity of the channel (Zúñiga et al., 2011).

Mechanistically, two possibilities might explain the phenotype of heterodimers (combinations of WT and G312A or WT and $\Delta \mathrm{pCt}$; cooperative concerted gating properties) in TREK-2: first, the movements of WT subunit might lead to similar movements of the mutated or deleted subunit, so that the phenotype of heterodimer is analogous to those of WT-WT. Second, the movements of WT subunit are sufficient to manipulate the pore of TREK-2 to control the efflux of potassium ions. Although the negative gating subunits are not able to control gating, they might be necessary to maintain the normal structure of the pore. That is, they might exert structural function. Although we tend to believe that the second possibility rarely occurs, our data and current crystallographic evidence are not capable of excluding it completely.

Although both G312 and pCt domain gate TREK-2 in a intersubunit cooperative fashion in both 2 -APB and $\mathrm{pH}_{\mathrm{o}}$ pathways, the nature of such cooperativity might depend on the specific mutation or stimuli used to the gating process. A recent study reveal that both sensors of ruthenium red (D135), located on the extracellular side, contribute to its inhibitory effect on TREK-2 (Braun et al., 2015), implicating that TREK-2 might also adopt intersubunit sequential or independent mechanism to gate the pore. Interestingly, although one $\mathrm{Ct}$ is sufficient to gate the pore in the regulations by extracellular acidification and $\mathrm{G}$ protein coupled receptors in TREK-1 (Sandoz et al., 2012), which suggests positive cooperativity, it seems that $\mathrm{Ct}$ also gates the pore via a negatively cooperative mechanism, as demonstrated by other group (Bagriantsev et al., 2012). Thus, data related to the gating mechanisms of channel with concatenated heteromeric channels should be carefully interpreted, as that suggested in the C-type inactivation and drug binding studies of hERG1 channels (Wu et al., 2014a, 2015a,b).

Since the movements of M4 and pCt domain gate the pore in both 2-APB and $\mathrm{pH}_{\mathrm{o}}$ pathways in a completely concerted cooperative manner, we further determined whether cis- or trans-type mechanism mediates G312 and pCt in the crosstalk between SF gate and cytosolic pCt domain in TREK-2. According to our results, further introduction of $\triangle \mathrm{pCt}$ into the latter subunit of G312A-WT and G312A-G312A blunted

\section{REFERENCES}

Acosta, C., Djouhri, L., Watkins, R., Berry, C., Bromage, K., and Lawson, S. N. (2014). Trek2 expressed selectively in ib4-binding c-fiber nociceptors hyperpolarizes their membrane potentials and limits spontaneous pain. J. Neurosci. 34, 1494-1509. doi: 10.1523/JNEUROSCI.4528-13.2014 their responses to both 2-APB and $\Delta \mathrm{pH}_{\mathrm{o}}$ (Figure 5), suggesting that the bidirectional signal transduction between $\mathrm{pCt}$ domain and SF gate via M4 is not able to occur across two subunits. Namely, they gate TREK-2 through a cis-type mechanism, with transduction along one single subunit. Similar mechanism has also been found in TREK-1 when regulating temperature response (Bagriantsev et al., 2012).

Mechanistically, the energy gained from occupancy by a single 2-APB or alkalization of a single $\mathrm{pH}_{\mathrm{o}}$ sensor might be sufficient to manipulate channel gating, and allow the signals be transduced along the subunit in TREK-2. A possible interpretation is that, another subunit might only exert structural functions at least in these stimuli-induced gating, implicating the function of the channel is not associated with its dimeric status. Consistently, several studies have reported that some K2Ps do not require the disulfide bond for formatting homodimer or their functions (Patel et al., 2000; Niemeyer et al., 2003; Hwang et al., 2014).

In summary, our data suggest that the WT subunit exerts fully dominant roles to the mutated one by tagging one of the subunit types with conductance mutations to report subunit stoichiometry. And further incorporating conductance mutations into the heterodimeric channels suppress both pathways. These results support that intersubunit concerted cooperative and cis-type mechanism control the pore gating of TREK-2 during 2-APB and $\Delta \mathrm{pH}_{\mathrm{o}}$-induced transductions.

\section{AUTHOR CONTRIBUTIONS}

$\mathrm{X}-\mathrm{YM}, \mathrm{R}-\mathrm{GZ}$, and X-LW conceived the study, engineered mutant constructs, performed electrophysiological experiments, collected and analyzed data, and wrote the manuscript. PP and X-YL performed electrophysiological experiments. H-TY constructed mutant channels. J-PX and J-QZ edited the manuscript.

\section{ACKNOWLEDGMENTS}

We thank Dr. Miaozong Wu (Center for Diagnostic Nanosystems, Marshall University) for kindly reading and revising the manuscript. This work was supported by grants from the National Science Foundation of China Grants (31200797), the National Major Scientific and Technological Special Project for "Significant New Drug Development" (2014ZX09507-003), the National Integrated Drug Discovery Technology Platform Foundation of China (2012ZX09301003001), the Natural Science Foundation of Fujian Province of China (No. 2016J05203) and Guangdong Natural Science Foundation (No.2016A030310371). 
Braun, G., Lengyel, M., Enyedi, P., and Czirják, G. (2015). Differential sensitivity of trek-1, trek-2 and traak background potassium channels to the polycationic dye ruthenium red. Br. J. Pharmacol. 172, 1728-1738. doi: 10.1111/bph.13019

Clarke, C. E., Veale, E. L., Wyse, K., Vandenberg, J. I., and Mathie, A. (2008). The m1p1 loop of task3 k2p channels apposes the selectivity filter and influences channel function. J. Biol. Chem. 283, 16985-16992. doi: 10.1074/jbc. M801368200

Cohen, A., Ben-Abu, Y., Hen, S., and Zilberberg, N. (2008). A novel mechanism for human k2p2.1 channel gating. Facilitation of c-type gating by protonation of extracellular histidine residues. J. Biol. Chem. 283, 19448-19455. doi: 10. 1074/jbc.M801273200

daCosta, C. J., Free, C. R., and Sine, S. M. (2015). Stoichiometry for alphabungarotoxin block of alpha7 acetylcholine receptors. Nat. Commun. 6:8057. doi: $10.1038 /$ ncomms 9057

Deng, P. Y., Xiao, Z., Yang, C., Rojanathammanee, L., Grisanti, L., Watt, J., et al. (2009). Gaba(b) receptor activation inhibits neuronal excitability and spatial learning in the entorhinal cortex by activating trek- $2 \mathrm{k}^{+}$channels. Neuron 63 , 230-243. doi: 10.1016/j.neuron.2009.06.022

Enyedi, P., and Czirják, G. (2010). Molecular background of leak k ${ }^{+}$currents: two-pore domain potassium channels. Physiol. Rev. 90, 559-605. doi: 10. 1152/physrev.00029.2009

Gagnon, D. G., and Bezanilla, F. (2009). A single charged voltage sensor is capable of gating the shaker k+ channel. J. Gen. Physiol. 133, 467-483. doi: 10.1085/jgp. 200810082

Gagnon, D. G., and Bezanilla, F. (2010). The contribution of individual subunits to the coupling of the voltage sensor to pore opening in shaker k channels: effect of ilt mutations in heterotetramers. J. Gen. Physiol. 136, 555-568. doi: 10.1085/jgp. 201010487

Hwang, E. M., Kim, E., Yarishkin, O., Woo, D. H., Han, K. S., Park, N., et al. (2014). A disulphide-linked heterodimer of twik-1 and trek-1 mediates passive conductance in astrocytes. Nat. Commun. 5:3227. doi: 10.1038/ ncomms 4227

Kang, D., and Kim, D. (2006). Trek-2 (k2p10.1) and tresk (k2p18.1) are major background $\mathrm{k}^{+}$channels in dorsal root ganglion neurons. Am. J. Physiol. Cell Physiol. 291, C138-C146. doi: 10.1152/ajpcell.00629. 2005

Koshland, D. E. Jr., Némethy, G., and Filmer, D. (1966). Comparison of experimental binding data and theoretical models in proteins containing subunits. Biochemistry 5, 365-385. doi: 10.1021/bi008 $65 \mathrm{a} 047$

Lesage, F., Terrenoire, C., Romey, G., and Lazdunski, M. (2000). Human TREK2, a $2 \mathrm{P}$ domain mechano-sensitive $\mathrm{k}^{+}$channel with multiple regulations by polyunsaturated fatty acids, lysophospholipids and, Gs, Gi, and Gq protein-coupled receptors. J. Biol. Chem. 275, 28398-28405. doi: 10.1074/jbc. M002822200

Li, Z. B., Zhang, H. X., Li, L. L., and Wang, X. L. (2005). Enhanced expressions of arachidonic acid-sensitive tandem-pore domain potassium channels in rat experimental acute cerebral ischemia. Biochem. Biophys. Res. Commun. 327, 1163-1169. doi: 10.1016/j.bbrc.2004.12.124

Ma, X. Y., Yu, J. M., Zhang, S. Z., Liu, X. Y., Wu, B. H., Wei, X. L., et al. (2011). External ba2+ block of the two-pore domain potassium channel trek1 defines conformational transition in its selectivity filter. J. Biol. Chem. 286, 39813-39822. doi: 10.1074/jbc.M111.264788

Meisel, E., Dvir, M., Haitin, Y., Giladi, M., Peretz, A., and Attali, B. (2012). Kcnq1 channels do not undergo concerted but sequential gating transitions in both the absence and the presence of kcnel protein. J. Biol. Chem. 287, 34212-34224. doi: 10.1074/jbc.M112.364901

Monod, J., Wyman, J., and Changeux, J. P. (1965). On the nature of allosteric transitions: a plausible model. J. Mol. Biol. 12, 88-118. doi: 10.1016/s00222836(65)80285-6

Niemeyer, M. I., Cid, L. P., Peña-Munzenmayer, G., and Sepúlveda, F. V. (2010). Separate gating mechanisms mediate the regulation of $\mathrm{k} 2 \mathrm{p}$ potassium channel task-2 by intra- and extracellular ph. J. Biol. Chem. 285, 16467-16475. doi: 10. 1074/jbc.M110.107060

Niemeyer, M. I., Cid, L. P., Valenzuela, X., Paeile, V., and Sepúlveda, F. V. (2003). Extracellular conserved cysteine forms an intersubunit disulphide bridge in the kcnk5 (task-2) k+ channel without having an essential effect upon activity. Mol. Membr. Biol. 20, 185-191. doi: 10.1080/0968768031000084181
Patel, A. J., Maingret, F., Magnone, V., Fosset, M., Lazdunski, M., and Honoré, E. (2000). Twik-2, an inactivating 2 p domain $\mathrm{k}^{+}$channel. J. Biol. Chem. 275, 28722-28730. doi: 10.1074/jbc.M003755200

Pathak, M., Kurtz, L., Tombola, F., and Isacoff, E. (2005). The cooperative voltage sensor motion that gates a potassium channel. J. Gen. Physiol. 125, 57-69. doi: 10.1085/jgp.200409197

Pereira, V., Busserolles, J., Christin, M., Devilliers, M., Poupon, L., Legha, W., et al. (2014). Role of the trek2 potassium channel in cold and warm thermosensation and in pain perception. Pain 155, 2534-2544. doi: 10.1016/j.pain.2014. 09.013

Perutz, M. F. (1989). Mechanisms of cooperativity and allosteric regulation in proteins. Q. Rev. Biophys. 22, 139-237. doi: 10.1017/s0033583500 003826

Rivera-Pagán, A. F., Rivera-Aponte, D. E., Melnik-Martínez, K. V., ZayasSantiago, A., Kucheryavykh, L. Y., Martins, A. H., et al. (2015). Up-regulation of trek-2 potassium channels in cultured astrocytes requires de novo protein synthesis: relevance to localization of trek-2 channels in astrocytes after transient cerebral ischemia. PLoS One 10:e0125195. doi: 10.1371/journal.pone. 0125195

Sandoz, G., Douguet, D., Chatelain, F., Lazdunski, M., and Lesage, F. (2009). Extracellular acidification exerts opposite actions on trek1 and trek2 potassium channels via a single conserved histidine residue. Proc. Natl. Acad. Sci. U S A 106, 14628-14633. doi: 10.1073/pnas.09062 67106

Sandoz, G., Levitz, J., Kramer, R. H., and Isacoff, E. Y. (2012). Optical control of endogenous proteins with a photoswitchable conditional subunit reveals a role for trek1 in gaba(b) signaling. Neuron 74, 1005-1014. doi: 10.1016/j.neuron. 2012.04.026

Stansfeld, P. J., Grottesi, A., Sands, Z. A., Sansom, M. S., Gedeck, P., Gosling, M., et al. (2008). Insight into the mechanism of inactivation and ph sensitivity in potassium channels from molecular dynamics simulations. Biochemistry 47, 7414-7422. doi: 10.1021/bi800475j

Thomson, S. J., Hansen, A., and Sanguinetti, M. C. (2014). Concerted all-or-none subunit interactions mediate slow deactivation of human ether-a-go-go-related gene k+ channels. J. Biol. Chem. 289, 23428-23436. doi: 10.1074/jbc.M114. 582437

Tombola, F., Ulbrich, M. H., Kohout, S. C., and Isacoff, E. Y. (2010). The opening of the two pores of the hvl voltage-gated proton channel is tuned by cooperativity. Nat. Struct. Mol. Biol. 17, 44-50. doi: 10.1038/nsmb. 1738

Tytgat, J., and Hess, P. (1992). Evidence for cooperative interactions in potassium channel gating. Nature 359, 420-423. doi: 10.1038/359420a0

Wu, W., Gardner, A., and Sanguinetti, M. C. (2014a). Cooperative subunit interactions mediate fast c-type inactivation of herg1 k+ channels. J. Physiol. 592, 4465-4480. doi: 10.1113/jphysiol.2014.277483

Wu, W., Sachse, F. B., Gardner, A., and Sanguinetti, M. C. (2014b). Stoichiometry of altered herg1 channel gating by small molecule activators. J. Gen. Physiol. 143, 499-512. doi: 10.1085/jgp.201311038

Wu, W., Gardner, A., and Sanguinetti, M. C. (2015a). Concatenated herg1 tetramers reveal stoichiometry of altered channel gating by rpr-260243. Mol. Pharmacol. 87, 401-409. doi: 10.1124/mol.114.096693

Wu, W., Gardner, A., and Sanguinetti, M. C. (2015b). The link between inactivation and high-affinity block of herg1 channels. Mol. Pharmacol. 87, 1042-1050. doi: 10.1124/mol.115.098111

Xiao, Z., Cilz, N. I., Kurada, L., Hu, B., Yang, C., Wada, E., et al. (2014). Activation of neurotensin receptor 1 facilitates neuronal excitability and spatial learning and memory in the entorhinal cortex: beneficial actions in an Alzheimer's disease model. J. Neurosci. 34, 7027-7042. doi: 10.1523/JNEUROSCI.0408-14. 2014

Xiao, Z., Deng, P. Y., Rojanathammanee, L., Yang, C., Grisanti, L., Permpoonputtana, K., et al. (2009). Noradrenergic depression of neuronal excitability in the entorhinal cortex via activation of trek-2 $\mathrm{k}^{+}$channels. J. Biol. Chem. 284, 10980-10991. doi: 10.1074/jbc.M8067 60200

Yamamoto, Y., Hatakeyama, T., and Taniguchi, K. (2009). Immunohistochemical colocalization of trek-1, trek-2 and traak with trp channels in the trigeminal ganglion cells. Neurosci. Lett. 454, 129-133. doi: 10.1016/j.neulet.2009. 02.069 
Yifrach, O., Zandany, N., and Shem-Ad, T. (2009). Examining cooperative gating phenomena in voltage-dependent potassium channels: taking the energetic approach. Methods Enzymol. 466, 179-209. doi: 10.1016/S00766879(09)66008-0

Zandany, N., Ovadia, M., Orr, I., and Yifrach, O. (2008). Direct analysis of cooperativity in multisubunit allosteric proteins. Proc. Natl. Acad. Sci. U S A 105, 11697-11702. doi: 10.1073/pnas.08041 04105

Zhuo, R. G., Liu, X. Y., Zhang, S. Z., Wei, X. L., Zheng, J. Q., Xu, J. P., et al. (2015a). Insights into the stimulatory mechanism of 2-aminoethoxydiphenyl borate on trek-2 potassium channel. Neuroscience 300, 85-93. doi: 10.1016/j. neuroscience.2015.05.012

Zhuo, R. G., Peng, P., Liu, X. Y., Zhang, S. Z., Xu, J. P., Zheng, J. Q., et al. (2015b). The isoforms generated by alternative translation initiation adopt similar conformation in the selectivity filter in trek-2. J. Physiol. Biochem. 71, 601-610. doi: 10.1007/s13105-015-0422-Z

Zhuo, R. G., Peng, P., Liu, X. Y., Zhang, S. Z., Xu, J. P., Zheng, J. Q., et al. (2016). Allosteric coupling between proximal c-terminus and selectivity filter is facilitated by the movement of transmembrane segment 4 in trek- 2 channel. Sci. Rep. 6:21248. doi: 10.1038/srep21248

Zúñiga, L., Márquez, V., González-Nilo, F. D., Chipot, C., Cid, L. P., Sepúlveda, F. V., et al. (2011). Gating of a ph-sensitive $\mathrm{k}(2 \mathrm{p})$ potassium channel by an electrostatic effect of basic sensor residues on the selectivity filter. PLoS One 6:e16141. doi: 10.1371/journal.pone.0016141

Conflict of Interest Statement: The authors declare that the research was conducted in the absence of any commercial or financial relationships that could be construed as a potential conflict of interest.

Copyright (C) 2016 Zhuo, Peng, Liu, Yan, Xu, Zheng, Wei and Ma. This is an openaccess article distributed under the terms of the Creative Commons Attribution License (CC BY). The use, distribution and reproduction in other forums is permitted, provided the original author(s) or licensor are credited and that the original publication in this journal is cited, in accordance with accepted academic practice. No use, distribution or reproduction is permitted which does not comply with these terms. 